\title{
Some New Properties of Fuzzy Maximal Regular Open Sets
}

\author{
Mohammed M. Ali Al-Shamiri, Khaled A. Alzobydi
}

\begin{abstract}
A proper nonempty open subset $\boldsymbol{\mu}$ of a fuzzy topological space $(\boldsymbol{X}, \boldsymbol{\tau})$ is said to be a fuzzy maximal regular open set, if any regular open set which contains $\boldsymbol{\mu}$ is $\mathbf{1}_{\boldsymbol{X}}$ or $\boldsymbol{\mu}$. The purpose of this paper is to study some new fundamental properties of fuzzy maximal regular open sets. The decomposition theorems for a fuzzy maximal regular open set are investigated. Notion and basic properties of radical of fuzzy maximal regular open sets are established, such as the law of fuzzy radical closure. Some new properties and characterization theorems of fuzzy maximal regular open set are achieved.
\end{abstract}

Keywords - Fuzzy Radical topology , Fuzzy Regular Radical, Fuzzy Maximal Regular Open Set, Maximal Regular Open Set, Regular Open Set.

\section{INTRODUCTION}

A $\mathrm{s}$ is known, a crisp set is defined by dividing individuals into two types - members and non-members. One notices a distinction between members and non-members of the class represented in a crisp set. But many of the sets and categories we normally use do not show this feature. Its borders seem ambiguous, and moving from one member to another seems gradual and not surprising. This is called a fuzzy set that offers ambiguity by eliminating the boundary between members of the acute class of non-members. In our lives more cases are often not clear and deterministic and cannot be accurately described. Such cases are characterized by ambiguity or inaccuracy that cannot be answered only by yes or no. Lutfi Zadeh [19] in 1965 presented the idea of a fuzzy set to describe mathematically in highly abstract and solving these problems gave a certain degree of membership to each member of a given set.

This is actually the basic setting of the mysterious fuzzy set theory. Specify a fuzzy set as a generalization for the characteristic function of the set where the degree of element membership is more general than "Yes" or "No".

A mathematically fuzzy set can be determined by assigning to each potential in dividual in the universe of discourse a value that represents its grade of membership in the fuzzy set.

Mohammed M. Ali Al-Shamiri is with the Department of Mathematics, Faculty of Science and Arts, Mahayil Asir, King Khalid University, K.S.A., Saudi Arabia and in the Department of Mathematics and computer, Faculty of science, Ibb University, Ibb, Yemen. (e-mail: mal-shamiri@kku.edu.sa).

Khaled A. Alzobydi is with the Department of Mathematics, Faculty of Science and Arts, Mahayil Asir, King Khalid University, K.S.A., Saudi Arabia.
This grade corresponds to the degree of compatibility of this individual with the concept represented by the fuzzy set. Membership scores are often represented by a number of real values ranging in the closed interval period between 0 and 1 . The research activities in this area and related areas have found applications in many branches of Science and other in life fields as computer science and engineering. C. L. Chang [4], in 1968 introduced and studied the concept of fuzzy topological spaces as a generalization of topological spaces. Follow him many researchers like R.H. Warren ( [17] -[18]) K. K. Azad ( [1]-[2]) G. Balasubramanian and P. Sundaram [3], S. R.Malghan and S. S. Benchalli ( [7]-[8] ), M. N. Mukerjee and B. Ghosh [9], A. Mukherjee [10], J. A. Goguen [5] and many others, all of them contributed to the development of mysterious topological spaces in successive years. The minimal open sets and maximal open sets was introduced by 2001-2003, Nakaoka and Oda ([11]-[12] -[13]), which are subclasses of open sets. K. K. Azad [1] defined fuzzy regular open sets in fuzzy topological spaces in the year1981. Thereafter, Mathematicians gave in several papers in different and interesting new open sets. In this paper, we will introduce and study some new fundamental properties of fuzzy maximal regular open sets. And we will be investigate the decomposition theorems for a fuzzy maximal regular open set. Also we will be establish nation and basic properties of radical of fuzzy maximal regular open sets, which is a law of fuzzy radical closure. We will be Achieve some basic and new properties and characterization theorems of fuzzy maximal regular open sets.

\section{PRELIMINARIES}

We begin with a few basic definitions and results as our prerequisites.

Definition 1.2: [15] Let $X$ be a non empty set and I be the closed interval $[0,1]$. Denoted by $\mathrm{I}^{\mathrm{X}}$ to the collection of all functions from $\mathrm{X}$ intoI, a fuzzy set $\mu$ on $\mathrm{X}$ is a member of $\mathrm{I}^{\mathrm{X}}$. Its support denoted by $\operatorname{supp} \mu$, is the crisp set $\{\mathrm{x} \in \mathrm{X}$ : $\mu(x)>0\}$. A fuzzy set with a singleton as its support is called a fuzzy point, denoted by $\mathrm{x}_{\mathrm{t}}$, and defined as,

$$
\mathrm{x}_{\mathrm{t}}(\mathrm{z})= \begin{cases}\mathrm{t}, & \text { for } \mathrm{z}=\mathrm{x} \\ 0 & \text { otherwise. }\end{cases}
$$

Here we note that a fuzzy singleton $x_{t}$ belongs to a fuzzy set $\mu$ i.e. $\left(x_{t} \in \mu\right)$ iff $t \leq \mu(x)$. 
Definition 2.2: [15] Let $X$ be a set and $\tau$ a family of fuzzy subsets of $X$.Then $\tau$ is called fuzzy topology on $X$ if satisfies the following conditions:

(1) $1_{\phi}, 1_{\mathrm{X}} \in \tau$.

(2) If $\lambda, \mu \in \tau$, then $\lambda \wedge \mu \in \tau$.

(3) If $\lambda_{i} \in \tau$, for each $i \in \Lambda$, then $\bigvee \lambda_{i} \in \tau$.

The ordered pair $(\mathrm{X}, \tau)$ is called a fuzzy topological space (FTS, for short).Moreover, the members of $\tau$ is said to be the fuzzy open sets and their complements are said to be fuzzy closed sets.

Definition 3.2: [14]: A fuzzy set $\lambda$ in a FTS $X$ is called a nbd. of a fuzzy point $x_{t}$ if and only if there exists a fuzzy open set $\mu$ in $X$ such that $x_{t} \in \mu \leq \lambda$

A nbd. $\lambda$ is said to be fuzzy open if and only if $\lambda$ itself is fuzzy open. A fuzzy point $x_{t} \in \bar{\lambda}$ if each neighborhood of $x_{t}$ intersection with $\lambda$.

Definition 4.2: [6] Let $(G, \delta)$ be a fuzzy topological space. The induced topology $\tau_{\delta}$ on the collection $\vartheta(G)$ of all fuzzy singletons of $G$ is defined as the topology generated by $\sigma=\left\{V_{\mu} \mid \mu \in \sigma\right\}$, where $V_{\mu}=\{p \in \vartheta(G) \mid p \in \mu\}$ and hence $\left(\vartheta(G), \tau_{\delta}\right)$ is called an induced topological space.

Definition 5.2: [1] A fuzzy subset $\lambda$ of FTS $X$ is said to be:

(1) fuzzy regular open set if $\operatorname{Int}(\operatorname{Cl}(\lambda))=\lambda$

(2) fuzzy regular closed set if $\operatorname{Cl}(\operatorname{Int}(\lambda))=\lambda$ or if $1_{X}-\lambda$ is fuzzy regular open set in $X$.

The class of all fuzzy regular open and fuzzy regular closed sets are respectively denoted by $F R O(X)$ and $F R C(X)$.

Definition 6.2: [16] Let $(X, \tau)$ be a fuzzy topological space. A nonzero fuzzy open subset $\mu$ of $X$ is said to be maximal fuzzy regular open set if $\mu \neq 1_{X}$ and for any fuzzy regular open set $\lambda$ in $\mathrm{X}$ with $\mu \leq \lambda$, then $\mu=\lambda$ or $\lambda=1_{\mathrm{X}}$.

Theorem 1.2[16].Union of arbitrary member of fuzzy maximal regular open sets is either fuzzy maximal regular open set or fuzzy whole set $1_{X}$.

\section{MAIN RESULTS}

Theorem 1.3: A non empty subset $\lambda$ of a fuzzy topological space $X$ is fuzzy maximal regular open set if and only if $1_{X}-\lambda$ is fuzzy minimal regular closed set.

Proof: Let $\lambda$ be a fuzzy maximal regular open set in $X$. Suppose $1_{\mathrm{X}}-\lambda$ is not a fuzzy minimal regular closed set in $\mathrm{X}$. Then there exists a fuzzy regular closed set $\mu$ in $X$ such that $1_{\phi} \neq \mu<1_{X}-\lambda$. That is $\lambda<1_{X}-\mu$ and $1_{X}-\mu$ is a fuzzy regular open set in $X$. This is contradiction to $\lambda$ is a fuzzy maximal regular open set in $X$. Therefore $1_{X}-\lambda$ is fuzzy minimal regular closed set in $\mathrm{X}$.

Conversely, let $1_{\mathrm{X}}-\lambda$ be a fuzzy minimal regular closed set in $X$. Suppose $\lambda$ is not a fuzzy maximal regular open set in $X$.
Then there exists a fuzzy regular open set $\mu$ such that $\lambda<$ $\mu \neq 1_{\mathrm{x}}$. That is $1_{\mathrm{x}}-\mu<1_{\mathrm{x}}-\lambda$ and $1_{\mathrm{x}}-\lambda$ is a fuzzy regular closed set in $\mathrm{X}$. This is contradiction and therefore $\lambda$ is a fuzzy maximal open set in $\mathrm{X}$.

Lemma1.3: Let $(X, \tau)$ be a fuzzy topological space.

i) If $\lambda$ is a fuzzy maximal regular open set and $\mu$ is a fuzzy open sets in $\mathrm{X}$, then

$\lambda \vee \mu=1_{\mathrm{X}}$ or $\mu<\lambda$.

ii) If $\lambda$ and $\mu$ are a fuzzy maximal regular open sets then $\lambda \vee \mu=1_{\mathrm{X}}$ or $\mu=\lambda$.

Proof: i) Let $\lambda$ be any fuzzy maximal regular open and $\mu$ be any fuzzy regular open sets in X. We suppose that $\lambda \vee \mu \neq 1_{X}$, then we want to prove that $\mu<\lambda$. Since $\lambda \vee \mu \neq 1_{X}$, then $\lambda<\lambda \vee \mu, \lambda \vee \mu$ is a fuzzy open and $\lambda$ is a fuzzy maximal regular open sets in $X$. Therefore $\lambda \vee \mu=1_{X}$ or $\lambda \vee \mu=\mu$. But $\lambda \vee \mu \neq 1_{X}$ then $\lambda \vee \mu=\mu \Rightarrow \mu<\lambda$.

ii) Since every fuzzy maximal regular open set is a fuzzy open set, it follows from (i) that $\mu<\lambda$ and $\lambda<\mu$. Therefore $\mu=\lambda$.

Theorem2.3: Let $\lambda, \mu$ and $\rho$ are fuzzy maximal regular open sets and $\lambda \neq \mu$ and if $\lambda \wedge \mu<\rho$, then either $\lambda=\rho$ or $\mu=\rho$.

Proof: Let $\lambda, \mu$ and $\rho$ are fuzzy maximal regular open sets and $\lambda \neq \mu$ and $\lambda \wedge \mu<\rho$.

If $\lambda \neq \rho$ then we want to prove that $\mu=\rho$. Now $\mu \wedge \rho=$ $\mu \wedge\left(\rho \wedge 1_{X}\right)=\mu \wedge[\rho \wedge(\lambda \vee \mu)$,

(by Lemma 1.3 (ii), $\lambda \vee \mu=1_{X}$.)

$=\mu \wedge[(\rho \wedge \lambda) \vee(\rho \wedge \mu)]$

$$
=(\mu \wedge \rho \wedge \lambda) \vee(\mu \wedge \rho \wedge \mu)
$$

$=(\mu \wedge \lambda) \vee(\mu \wedge \rho)$, (because $\lambda \wedge \mu<\rho$.) $=\mu \wedge(\lambda \vee \rho)=\mu \wedge 1_{X}=\mu$.

$\Rightarrow \mu \wedge \rho=\mu \Rightarrow \mu<\rho$. By definition of fuzzy maximal open sets, we get $\mu=\rho$.

Theorem 3.3: If $\lambda, \mu$ and $\rho$ are fuzzy maximal regular open sets such that $\mu \neq \lambda \neq \rho$, then $\lambda \wedge \mu \nless \lambda \wedge \rho$.

Proof: Suppose $\lambda \wedge \mu<\lambda \wedge \rho$, then we see that $(\lambda \wedge \mu) \vee(\mu \wedge \rho)<(\lambda \wedge \rho) \vee(\mu \wedge \rho)=(\lambda \vee \rho) \wedge$ $\mu<(\lambda \vee \mu) \wedge \rho=1_{X} \wedge \mu<1_{X} \wedge \rho=\mu<\rho$ And hence $\mu=\rho$ from the definition of fuzzy maximal open sets, but this contradiction. Therefore $\lambda \wedge \mu \nless \lambda \wedge \rho$.

Definition1.3: Let $\left\{\lambda_{i}: i \in I\right\}$ be a collection of all fuzzy maximal open set of a FTS X, $\mathcal{R}=\Lambda_{i \in I} \lambda_{i}$ is called radical of FTX.

Definition 2.3: Let $\left\{\lambda_{i}: i \in I\right\}$ be a collection of all fuzzy maximal regular open set of a FTS $X, \mathcal{R}=\Lambda_{i \in I} \lambda_{i}$ is called regular radical of FTX. 
Definition 3.3: Let $\left\{\lambda_{i}: i \in I\right\}$ be a collection of fuzzy maximal regular open set of a FTS X, $\mathcal{R}=\Lambda_{i \in I} \lambda_{i}$ is called regular radical of $\left\{\lambda_{i}: i \in I\right\}$.

Theorem 4.3: Let $\left\{\lambda_{i}: i \in I\right\}$ be a collection of fuzzy maximal regular open sets of a FTS $X$,if $|I|>2$ and $\lambda_{i} \neq \lambda_{j}$ for all

$i, j \in I$ and $i \neq j$,then:

1) $1_{X}-\Lambda_{i \in I \backslash\{j\}} \lambda_{i}<\lambda_{j}$.

2) $\Lambda_{i \in I \backslash\{j\}} \lambda_{i} \neq 1_{\phi}$.

3) $\lambda_{j}=\left(\bigwedge_{i \in I} \lambda_{i}\right) \vee\left(1_{X}-\bigwedge_{i \in I\{j\}} \lambda_{i}\right)$

Proof: 1) Let $j \in I$, since $\lambda_{i}$ and $\lambda_{j}$ are fuzzy maximal regular open sets and $i \neq j$, then $\lambda_{i} \vee \lambda_{j}=1_{X}$ and hence $1_{X}-\lambda_{j}<$ $\lambda_{i} \Rightarrow 1_{X}-\lambda_{j}<\Lambda_{i \in I \backslash\{j\}} \lambda_{i}$, therefore $1_{X}-\Lambda_{i \in I \backslash\{j\}} \lambda_{i}<\lambda_{j}$.

2) Let $j \in I$ and suppose $\Lambda_{i \in I \backslash\{j\}} \lambda_{i}=1_{\phi}$, then $\lambda_{i}=1_{X}$, this is a contradiction, and therefore $\Lambda_{i \in I \backslash\{j\}} \lambda_{i} \neq 1_{\phi}$.

3) From 1) we have $\left(\Lambda_{i \in I} \lambda_{i}\right) \vee\left(1_{X}-\Lambda_{i \in I\{j\}} \lambda_{i}\right)=$ $\left(\left(\Lambda_{i \in I\{j\}} \lambda_{i}\right) \wedge \lambda_{j}\right) \vee\left(1_{X}-\Lambda_{i \in I\{j\}} \lambda_{i}\right)=$

$\left[\left(\Lambda_{i \in I\{j\}} \lambda_{i}\right) \vee\left(1_{X}-\Lambda_{i \in I\{j\}} \lambda_{i}\right)\right] \vee\left[\lambda_{j} \vee\left(1_{X}-\Lambda_{i \in I\{j\}} \lambda_{i}\right)\right]=$ $1_{X} \wedge \lambda_{j}=\lambda_{j}$

Corollary1.3: Let $\left\{\lambda_{i}: i \in I\right\}$ be a collection of fuzzy maximal regular open set of a FTS X if $I$ is a finite set and $\lambda_{i} \neq \lambda_{j}$ for all $i, j \in I$ with $i \neq j$ and if $\Lambda_{i \in I} \lambda_{i}$ is a fuzzy closed set, then $\lambda_{j}$ is a fuzzy closed set for any element $j$ of $I$.

Proof: From 3) of theorem 4.3, we have, for any $j$ of $I$,

$\lambda_{j}=\left(\wedge_{i \in I} \lambda_{i}\right) \vee\left(1_{X}-\Lambda_{i \in I \backslash\{j\}} \lambda_{i}\right)=\left(\wedge_{i \in I}\right) \vee\left(\vee_{i \in I \backslash\{j\}}\left(1_{X}-\right.\right.$ $\left.\left.\lambda_{i}\right)\right)$, now $\vee_{i \in I \backslash\{j\}}\left(1_{X}-\lambda_{i}\right)$ is a fuzzy closed set because $I$ is finite and therefore $\lambda_{j}$ is a fuzzy closed set for any element $j$ of $I$.

Theorem 5.3: Let $\left\{\lambda_{i}: i \in I\right\}$ be a collection of fuzzy maximal regular open set of a FTS $X,|I| \geq 2$ and $\lambda_{i} \neq \lambda_{j}$ for all $i, j \in I$ with $i \neq j$, if radical $\left\{\lambda_{i}: i \in I\right\} \neq 1_{\phi}$, then $\left\{\lambda_{i}: i \in I\right\}$ is the set of all fuzzy maximal regular open set of FTS X.

Proof: suppose that $\lambda_{l}$ a fuzzy maximal regular open set such that $\lambda_{l} \notin\left\{\lambda_{i}: i \in I\right\}$, then $1_{\phi}=\wedge_{i \in I} \lambda_{i}=\wedge_{i \in(I \vee\{l\}) \backslash\{l\}} \lambda_{i} \neq 1_{\phi}$ that is contradiction and hence $\left\{\lambda_{i}: i \in I\right\}$ is the set of all fuzzy maximal regular open sets of FTS X.

Proposition 1.3: Let $\lambda, \mu$ be any fuzzy subset of FTS X, if $\vee \mu=1_{X}, \lambda \wedge \mu$ is fuzzy closed and $\lambda$ fuzzy open then $\mu$ is fuzzy closed.

Proof: Since $1_{X} \wedge \lambda<\mu$, then we have $(\lambda \wedge \mu) \vee\left(1_{X}-\lambda\right)=$ $\left(\lambda \vee\left(1_{X}-\lambda\right)\right) \wedge\left(\mu \vee\left(1_{X}-\lambda\right)=\mu \vee\left(1_{X}-\lambda\right)=\mu \quad\right.$ since $\lambda \wedge \mu$ and $1_{X}-\lambda$ are closed sets then $\mu$ is closed set.
Theorem 6.3: Let $\left\{\lambda_{i} i \in I\right\}$ be a collection of fuzzy maximal regular open set of a FTS X and $\lambda_{i} \vee \lambda_{j}=1_{X}$ for any $i$ and $j$ of $I$ such that $i \neq j$, if the regular radical of $\left\{\lambda_{i}: i \in I\right\}$ is a fuzzy closed set then the regular radical of $\left\{\lambda_{i}: i \in I, i \neq j\right\}$ is a fuzzy closed set.

Proof: Let $j$ be any element of $I$, for $i \neq j, \lambda_{i} \vee \lambda_{j}=1_{X}$ then we get $\lambda_{j} \vee\left(\Lambda_{i \in I \backslash\{j\}} \lambda_{i}\right)=\Lambda_{i \in I \backslash\{j\}}\left(\lambda_{i} \vee \lambda_{j}\right)=1_{X}$, since $\lambda_{j} \vee\left(\Lambda_{i \in I \backslash\{j\}} \lambda_{i}\right)=\Lambda_{i \in I} \lambda_{i}$ is fuzzy closed set. Therefore $\wedge_{i \in I \backslash\{j\}} \lambda_{i}$ is fuzzy closed set, which means, the regular radical of $\left\{\lambda_{i}: i \in I, i \neq j\right\}$ is a fuzzy closed set.

Corollary 2.3: Let $\left\{\lambda_{i}: i \in I\right\}$ be a collection of fuzzy maximal regular open sets of a FTS X and $\lambda_{i} \neq \lambda_{j}$ for any $i$ and $j$ of $I$ such that $i \neq j$,if regular radical of $\left\{\lambda_{i}: i \in I\right\}$ is a fuzzy closed set then the regular radical of $\left\{\lambda_{i}: i \in I, i \neq j\right\}$ is a fuzzy closed set.

Proof: Since $\lambda_{i} \neq \lambda_{j}$ for any $i$ and $j$ of $I$ such that $i \neq j$, we get $\lambda_{i} \vee \lambda_{j}=1_{X}$ by theorem 6.3 it follows that, $\Lambda_{i \in I \backslash\{j\}} \lambda_{i}$ is fuzzy closed set, which means, the regular radical of $\left\{\lambda_{i}: i \in I, i \neq j\right\}$ is fuzzy closed set.

Lemma 2.3: Let $\left\{\lambda_{i}: i \in I\right\}$ be a collection of fuzzy maximal regular open sets, if closure of radical $\left\{\lambda_{i}: i \in I\right\}=1_{X}$, then $C L\left(\lambda_{i}\right)=1_{X}$ for any $i \in I$.

Proof: From the given $1_{X}=C L\left(\wedge_{i \in I} \lambda_{i}\right)<C L\left(\lambda_{i}\right)$. It follows that $C L\left(\lambda_{i}\right)=1_{X}$.

Theorem7.3: Let $\left\{\lambda_{i}: i \in I\right\}$ be a collection of fuzzy maximal regular open sets, and $I$ is finite, if closure of radical $\left\{\lambda_{i}: i \in I\right\} \neq 1_{X}$, then there exists an element $i \in I$ such that $C L\left(\lambda_{i}\right)=\lambda_{i}$.

Proof: We assume that $\operatorname{CL}\left(\lambda_{i}\right)=1_{X}$ for any element $i \in$ $I$, since $\wedge_{i \in I \backslash\{j\}} \lambda_{i}$ is fuzzy open set for $j \in I$. And $\left.\wedge_{i \in I \backslash\{j\}} \lambda_{i}=\left(\wedge_{i \in I \backslash\{j\}} \lambda_{i}\right) \wedge 1_{X}\right)=$

$$
\left.\left(\left(\wedge_{i \in I \backslash\{j\}} \lambda_{i}\right) \wedge C L\left(\lambda_{i}\right)\right)<C L\left(\wedge_{i \in I \backslash\{j\}} \lambda_{i}\right) \wedge \lambda_{i}\right)=
$$

$C L\left(\wedge_{i \in I} \lambda_{i}\right) \Rightarrow C L\left(\wedge_{i \in I \backslash\{j\}} \lambda_{i}\right)<C L\left(\wedge_{i \in I} \lambda_{i}\right)$,

also we see that $\Lambda_{i \in I} \lambda_{i}<\Lambda_{i \in I \backslash\{j\}} \lambda_{i}$ and hence $C L\left(\wedge_{i \in I} \lambda_{i}\right)<$ $C L\left(\wedge_{i \in I \backslash\{j\}} \lambda_{i}\right)$ it follows $C L\left(\wedge_{i \in I} \lambda_{i}\right)=C L\left(\wedge_{i \in I \backslash\{j\}} \lambda_{i}\right)$ by mathematical induction on the element of $I$, we get $C L\left(\wedge_{i \in I} \lambda_{i}\right)=C L\left(\lambda_{i}\right)=1_{X}$, this is contradiction with the assumption radical $\left\{\lambda_{i}: i \in I\right\} \neq 1_{X}$. Therefore, there exist an element $i \in I$ such that $C L\left(\lambda_{i}\right)=\lambda_{i}$.

Theorem 8.3: Let $\left\{\lambda_{i}: i \in I\right\}$ be a collection of fuzzy maximal regular open sets, and $I$ is finite. Let $\Gamma$ be a subset of $I$ such that $C L\left(\lambda_{i}\right)=\lambda_{i}$ for any $\in I, C L\left(\lambda_{i}\right)=1_{X}$ for any $i \in I \backslash \Gamma$. Then closure radical of $\left\{\lambda_{i}: i \in I\right\}=\Lambda_{i \in \Gamma} \lambda_{i}$ and closure radical of $\left\{\lambda_{i}: i \in I\right\}=1_{X}$, if $\Gamma=\phi$. 
Proof: If $\Gamma=\phi$,by theorem 7.3 the result is holds ,if $\Gamma \neq \phi$, then we get $\wedge_{i \in \Gamma} \lambda_{I}=1_{X} \wedge\left(\wedge_{i \in \Gamma} \lambda_{i}\right)=\left(\Lambda_{i \in \Gamma} \lambda_{i}\right) \wedge$ $C L\left(\wedge_{i \in I \backslash \Gamma} \lambda_{i}\right)<C L\left(\left(\wedge_{i \in \Gamma} \lambda_{i}\right) \wedge\left(\wedge_{i \in I \backslash \Gamma} \lambda_{i}\right)=C L\left(\wedge_{i \in \mathrm{I}} \lambda_{i}\right)\right.$

by theorem 7.3 and fact that $\Lambda_{i \in \Gamma} \lambda_{i}$ is open set ,we have $C L\left(\wedge_{i \in \Gamma} \lambda_{i}\right)<C L\left(C L\left(\wedge_{i \in \mathrm{I}} \lambda_{i}\right)\right)=C L\left(\wedge_{i \in \mathrm{I}} \lambda_{i}\right)$ but $\wedge_{i \in \mathrm{I}} \lambda_{i}<$ $\wedge_{i \in \Gamma} \lambda_{i}$ and hence $C L\left(\Lambda_{i \in \mathrm{I}} \lambda_{i}\right)<C L\left(\wedge_{i \in \Gamma} \lambda_{i}\right)$. It follows that $C L\left(\wedge_{i \in \mathrm{I}} \lambda_{i}\right)=C L\left(\Lambda_{i \in \Gamma} \lambda_{i}\right)$. Since $\lambda_{i}$ is closed set for any $i \in$

$\Gamma$,then closure radical of $\left\{\lambda_{i}: i \in I\right\}=\Lambda_{i \in \Gamma} \lambda_{i}$.

\section{REFERENCES}

[1] K. K. Azad. On fuzzy semi continuity, fuzzy almost continuity and fuzzy weakly continuity, J.Math. Anal. Appl., No. 1, pp.14 - 32, 82 (1981).

[2] K. K. Azad. Fuzzy Hausdorff spaces and fuzzy perfect mappings. J. Math. Anal. Appl. ,pp.297 - 305, 82 (1981).

[3] G. Balasubramanian and P. Sundaram. On some generalizations of fuzzy continuous functions, Fuzzy Sets and Systems , No.1,pp.93- 100, 86(1997).

[4] C. L. Chang. Fuzzy topological spaces., J. Math. Anal. Appl., pp.182 190, 24 (1968).

[5] J. A. Goguen. The fuzzy Tychonoff theorem. J. Math. Anal. Appl., No. 3, pp.734-742, 43 (1973).

[6] Lakshmana , V. Gomathi Nayagam, Geetha Sivaraman, Induced topology on fuzzy singletons, Far East Journal of Applied Mathematics, pp.189-198, 32 (2008).

[7] S. R. Malghan and S. S. Benchalli. Open maps, closed maps and local compactness in fuzzy topological spaces. J. Math. Anal. Appl., No. 2, 338-349, 99 (1984).

[8] S. R. Malghan and S. S. Benchalli. On fuzzy topological spaces. Glas. Mat. Ser. III Vol. 16(36), no. 2, 313-325, (1981).

[9] A. Mukerjee. On fuzzy completely semi continuous and weakly completely semicontinuous functions. Indian J. Pure Appl. Math., No. 191-197, 29 (1998).

[10] M. N. Mukherjee and B. Ghosh. Some stronger forms of fuzzy continuous mappings on fuzzy topological spaces. Fuzzy Sets and Systems, 375 - 387, 38 (1990).

[11] F. Nakoda and N. Oda. Some properties of maximal open sets. Int. J. Math. Math. Sci., pp.1331-1340,21 (2003).

[12] F. Nakoda and N. Oda. Some applications of minimal open sets. Int. J. Math. Math. Sci., No. 8, pp.471 - 476, 27 (2001).

[13] F. Nakaoka and N. Oda. On Minimal closed sets Proceedings of Topological spaces,. Theory and its Applications, 19 - 21, 5 (2003).

[14] Nayagam,V.L.G.D.Gauld,G.Venkateshwarian Sivaraman, "Strong Fuzzy Topological Group" New Zland Journal of mathematics, pp.187195, 38 (2008).

[15] Pu, P. M. and Y. M. Liu, Fuzzy topology I. Neighborhood structure of a fuzzy point and Moore- Smith convergence, J. Math. Anal. Appl,pp. 571-599, 76 (1980).

[16] B. Vijayalakshmi, On Fuzzy maximal regular semi-open set and maps in fuzzy topological spaces, Scientia Magna, Vol.12, No.1, pp.92-106, (2017)

[17] R. H. Warrren, Continuity of mappings of fuzzy topological spaces. Notices Amer. Math. Soc. A-451,21 (1974).

[18] R. H. Warren. Neighborhoods, bases and continuity in topological spaces. Rocky Mountain J. Math., No.3, pp.459 - 470, 8 (1978).

[19] L. A. Zadeh. Fuzzy sets. Information and Control. 8 , pp.338 - 353,8 (1965). 